Revista Brasil. Bot., V.34, n.3, p.389-402, jul.-set. 2011

\title{
Dinâmica espaço-temporal de Melocactus ernestii subsp. ernestii (Cactaceae) no Nordeste do Brasil
}

\author{
FREDERIC MENDES HUGHES ${ }^{1,3}$, MARCELINO DE LA CRUZ ROT ${ }^{2}$, \\ ROBERTO LISBOA ROMÃO ${ }^{1}$ e MARINA SIQUEIRA DE CASTRO ${ }^{1}$
}

(recebido: 17 de dezembro de 2010; aceito: 28 de julho de 2011)

\begin{abstract}
Spatio-temporal dynamics of Melocactus ernestii subsp. ernestii (Cactaceae) in northwestern Brazil): Melocactus ernestii Vaupel ssp. ernestii occurs in discontinuous outcrops in eastern Brazil. We investigated the spatio-temporal pattern of mortality events and survival of seedlings of $M$. ernestii relationship with plants and perennials, from the use of plots, assigned with the aid of measuring tapes, which were taken the Cartesian coordinates of the centroid of the stages of development, with subsequent analysis of point patterns based on Ripley's K function. Were mapped in plots 686 individuals of M. ernestii. The spatial pattern varied depending on the scale of the development stage and degree of weathering of the outcrops. The recruitment events and mortality were spatially structured and influenced by seasonal pulses of rainfall. Seedlings emerged ca. $50 \mathrm{~cm}$ from the centroid of adults. From the 136 seedlings monitored for 18 months, $84 \%$ died. The results showed that microsites initially effective in the maintenance of seed germination may not be effective in the establishment of seedlings. The magnitude of biotic interactions in stressful environments is species-specific and indicates that a positive association between adult plants and seedling survival is not consistent over time. This type of modeling population allows a better understanding of the role of positive and negative interactions as forces community structure, and may help to understand the complexity of biotic interactions in severe environmental gradients.
\end{abstract}

Key words - independent labeling model, recruitment, Ripley's K function, spatial structure

RESUMO - (Dinâmica espaço-temporal de Melocactus ernestii subsp. ernestii (Cactaceae) no Nordeste do Brasil) Melocactus ernestii Vaupel subsp. ernestii ocorre em afloramentos rochosos descontínuos no leste do Brasil. O presente estudo investigou o padrão espaço-temporal dos eventos de mortalidade e sobrevivência de plântulas da $M$. ernestii e a relação com as plantas perenes, a partir da utilização de parcelas, alocadas com auxílio de trenas, onde foram tomadas as coordenadas cartesianas do centróide dos estádios de desenvolvimento, com posterior análises de padrões de ponto baseado na função K de Ripley. Foram mapeados nas parcelas 686 indivíduos da M. ernestii. O padrão espacial variou em função da escala, do estádio de desenvolvimento e do grau de intemperização dos afloramentos. Os eventos de recrutamento e mortalidade foram espacialmente estruturados e influenciados por pulsos de precipitação sazonais. As plântulas emergiram a ca. $50 \mathrm{~cm}$ do centróide dos adultos. Das 136 plântulas monitoradas em 18 meses, $84 \%$ morreram. Os resultados mostraram que microsítios inicialmente eficazes na manutenção de sementes e promotores da germinação não necessariamente podem ser eficazes para o estabelecimento das plântulas. A magnitude das interações bióticas em ambientes estressantes é espécieespecífica e indica que associação positiva entre plantas adultas e sobrevivência de plântulas não é consistente com o tempo. Este tipo de modelagem populacional permite melhor compreensão sobre o papel das interações positivas e negativas como forças estruturadoras de comunidades e pode ajudar a entender a complexidade das interações bióticas em gradientes ambientais severos.

Palavras-chave - estrutura espacial, função K de Ripley, modelo de marcas independentes, recrutamento

\section{Introdução}

O gênero Melocactus Link \& Otto está inserido na família Cactaceae, uma das mais distintas famílias de plantas do Novo Mundo (Hunt et al. 2006), e é constituído de um total de 36 espécies (Anderson 2001). O centro de

1. Universidade Estadual de Feira de Santana, Departamento de Ciências Biológicas, 44031-460 Feira de Santana, BA, Brasil.

2. Universidade Politécnica de Madrid, Departamento de Biologia de Plantas, 28040 Madrid, Espanha.

3. Autor para correspondência: fmhughes@ufmg.br diversidade foi proposto para o Estado da Bahia (Rizzini 1982, Ritz et al. 2007), com 18 táxons endêmicos de um total de 22 espécies e subespécies reconhecidas para o estado (Taylor \& Zappi 2004), muitas destas (ca. 45\%) ameaçadas de extinção (IUCN 2009).

A distribuição e demografia dos cactos é afetada por múltiplos fatores que interatuam em diferentes escalas de espaço e tempo (Flores et al. 2004, Colaço et al. 2006, Méndez et al. 2006, Munguía-Rosas \& Sosa 2008, Peters et al. 2008). Essas interações espaço-temporais são dinâmicas e podem mudar dependendo do estádio de desenvolvimento e da história de vida da planta ou da 
severidade abiótica e que geram flutuação na densidade populacional em função tanto de processos dependentes da densidade quanto estocásticos ou independentes da densidade (Ruedas et al. 2000, Chu et al. 2008, De la Cruz et al. 2008).

Estudos envolvendo Cactaceae mostram que plantas adultas e arbustos favorecem a germinação e estabelecimento de novos indivíduos de cactos na população pelas modificações nas propriedades do solo e condições microclimáticas, i.e. nurse plants ou plantas berçário (Flores \& Jurado 2003, López \& Valdivia 2007, Larrea-Alcázar \& Soriano 2008). Embora associações entre cactos e plantas perenes tenham sido documentadas (Franco \& Nobel 1989, Valiente-Banuet \& Ezcurra 1991, Arriaga et al. 1993, Reyes-Olivas et al. 2002, GodínezÁlvarez \& Valiente-Banuet 2004, Godínez-Álvarez et al. 2005, Larrea-Alcázar \& Soriano 2008), a importância destas associações na distribuição espacial de cactos globosos é pouco estudada (Mandujano et al. 2002, Reyes-Olivas et al. 2002, Larrea-Alcázar \& Soriano 2008).

Algumas espécies de cactos toleram o estresse imposto pela radiação solar direta e se estabelecem em espaços abertos, uma vez que o recrutamento também é facilitado por elementos do microrrelevo tais como pedras, buracos ou fendas, denominados de nurse objects (Valiente-Banuet \& Ezcurra 1991, Reyes-Olivas et al. 2002, Flores \& Jurado 2003, Munguía-Rosas \& Sosa 2008). Cactos globulares ocorrem em microhabitats sob arbustos e também são capazes de se estabelecer em áreas abertas, sugerindo que a associação positiva entre cactos e plantas perenes é facultativa (Mandujano et al. 2002, López \& Valdivia 2007, Larrea-Alcázar \& Soriano 2008).

O cacto globoso Melocactus ernestii Vaupel subsp. ernestii ocorre exclusivamente no leste do Brasil desde o NE de Minas Gerais até os Brejos de Altitude nos estados de Pernambuco e Paraíba, com populações disjuntas em inselbergs gnáissicos e rochas cristalinas e areníticas circundadas por matriz de Caatinga arbórea consistindo predominantemente de vegetação xerófila (Taylor \& Zappi 2004).

O presente trabalho focou no estudo da demografia, associação espacial e temporal entre $M$. ernestii em diferentes estádios de desenvolvimento e entre os cactos e as plantas perenes para determinar níveis da estrutura espacial e os eventos de recrutamento e mortalidade. As hipóteses levantadas foram: (1) Flutuações na densidade populacional de $M$. ernestii são determinadas por eventos sazonais de precipitação e não são regulados por processos dependentes da densidade, (2) Há uma mudança na estrutura espacial dos diferentes estádios de desenvolvimento de M. ernestii, (3) As plantas adultas de M. ernestti facilitam o recrutamento e estabelecimento de suas plântulas, (4) Plântulas de $M$. ernestii estão associadas positivamente com as plantas perenes, (5) Os eventos de sobrevivência e mortalidade de $M$. ernestii estão estruturados espacialmente.

\section{Material e métodos}

Área e espécie de estudo - M. ernestii Vaupel subsp. ernestii é amplamente distribuída no leste do Brasil (Taylor \& Zappi 2004). É uma planta suculenta, com forma globular e com um cefálio terminal cilíndrico em adultos. A floração e frutificação ocorrem de forma contínua ao longo do ano, mas com picos sazonais da produção; o sistema reprodutivo é misto, representado por baixos níveis de autogamia e elevada produção de frutos e sementes por polinização cruzada; os beija-flores são os principais vetores de pólen; frutos e sementes são dispersos por lagartos territoriais, como, por exemplo, Tropidurus semitaeniatus Spix, 1825 (Fonseca et al. 2008, Hughes 2009).

O estudo foi conduzido em um fragmento de floresta estacional decidual, dentro dos domínios morfoclimáticos do bioma Caatinga (IBGE 1993, Juárez \& Liu 2001), com enclaves de afloramentos rochosos areníticos com distintos níveis de desgaste, no município de Nova Itarana, Bahia, Brasil (12 $57^{\prime}$ S, 39 $9^{\circ} 55^{\prime} \mathrm{W}$; 525 m). Durante o mapeamento dos três afloramentos encontrados, as parcelas foram categorizadas com base nos percentuais de solo: $<25 \%$, ligeiramente intemperizado, entre $25 \%$ e $50 \%$ moderadamente intemperizado e $>50 \%$, muito intemperizado; $1 / 3$ das parcelas foram enquadradas em uma ou outra categoria mencionada perfazendo $43,9 \%$ de solo na área de estudo. A tipologia climática da região varia de semi-árido e subúmido a seco, com temperatura média de $25^{\circ} \mathrm{C}$ e a precipitação média anual é de aproximadamente $700 \mathrm{~mm}$; a estação chuvosa ocorre de novembro a abril (Inmet 2009). Esta região é uma área prioritária para conservação da biodiversidade do bioma caatinga, apresentando fragmentos de mata seca e afloramentos rochosos preservados com elevado número de endemismos (MMA 2004).

Padrão espacial - Foram estabelecidas sete parcelas contíguas de $10 \times 10 \mathrm{~m}$ e duas outras parcelas em afloramentos menores: uma de $10 \times 10 \mathrm{~m}$ e outra de $15 \times 15 \mathrm{~m}$ (Parcela "B") englobando totalmente os afloramentos. Dentro de cada parcela foram registradas as coordenadas do centróide de cada planta em relação aos eixos das parcelas e também os seus diâmetros. Os indivíduos foram classificados de acordo com o tamanho e morfologia de plântulas $(<50 \mathrm{~mm}$ de diâmetro), juvenis ( $\geq 50 \mathrm{~mm}$ ) e adultos (com cefálio). As nove parcelas foram agrupadas para avaliar o padrão espacial em uma ampla área de $40 \times 40 \mathrm{~m}$ - englobando as sete parcelas contíguas, $70 \times 70 \mathrm{~m}-$ a parcela anterior mais a de 
$15 \times 15 \mathrm{~m}$ e $90 \times 90 \mathrm{~m}-$ todas as nove parcelas. Ajustaramse os eixos cartesianos $(\mathrm{x} ; \mathrm{y})$ das parcelas agrupadas para se ter valores dos pares coordenados compreendidos na mesma área amostral evitando duplicidade de pontos e compondo desse modo uma matriz de estrato arbóreo com ilhas de afloramentos.

Aspectos demográficos - Foi selecionada aleatoriamente uma parcela para acompanhar todos os indivíduos de $M$. ernestii em 18 meses (censo mensal de julho de 2007 a dezembro de 2008). Foram registradas as coordenadas de todas as plantas perenes encontradas e os casos de recrutamento, crescimento, mortalidade e sobrevivência de $M$. ernestii.

Análises estatísticas - Após constatação da normalidade e homogeneidade pelos testes Shapiro-Wilk e Bartlett a partir dos resíduos na ANOVA, foram realizados testes comparativos das médias (Tukey, $P<0,05$ ) entre o grau de intemperismo do afloramento rochoso (i.e. categorias relativas aos percentuais de solo nas parcelas estudadas) e o número de indivíduos em cada estádio de desenvolvimento (i.e. plântula, juvenil, adulto e todas as categorias em conjunto). A hipótese nula estabelecida foi a de ausência de dependência dos estádios da espécie ao espaço colonizado.

Aspectos demográficos - Foram conduzidas análises de correlação de Spearman e testes de Wilcoxon $\left(\mathrm{H}_{0}: \mathrm{m}_{+}=\mathrm{m}_{-}\right)$ entre a sobrevivência e a mortalidade total mensal de plântulas de $M$. ernestii e a precipitação média mensal, temperatura e umidade (Inmet 2009), para verificar as interrelações entre variáveis climáticas e demográficas e se as flutuações na densidade populacional ocorriam além desses níveis (i.e. primeira hipótese).

Padrão espacial de distribuição - O padrão de distribuição espacial nos estádios de desenvolvimento de $M$. ernestii em separado (análise univariada) e entre as fases (análise bivariada) (i.e. segunda hipótese) foi determinado utilizando a função K de Ripley (Ripley, 1977). A função K calcula todas as distâncias entre todas as plantas em um mapa de pontos para investigar o padrão espacial. De maneira simplificada, o processo consiste de um círculo de raio t centrado em cada planta, onde o número de vizinhos presentes dentro da área do círculo é contado: variando o raio é possível detectar o padrão espacial em escalas diferentes. Esta flexibilidade na escala de distâncias é que difere substancialmente a função K de outras ferramentas usadas (De la Cruz et al. 2008).

Para facilitar a visualização e interpretação dos resultados, foi utilizada a função $\mathrm{L}(\mathrm{t})=(\mathrm{K}(\mathrm{t}) / \pi)^{1 / 2}-\mathrm{t}$, uma forma linearizada da função $\mathrm{K}$ como sugerido por Ripley (1977). Para avaliar a segunda hipótese (i.e. diferença entre o padrão espacial das fases de desenvolvimento), a hipótese nula de Completa Aleatoriedade Espacial (CSR) para a função univariada foi assumida e testada através de simulações de Monte Carlo, construindo intervalos de confiança através dos maiores e menores valores da Função K para cada simulação e para cada escala de distância.
Para a análise de padrões entre os pares de estádios de desenvolvimento de $M$. ernestii e entre as plantas perenes, foi empregado um estimador bivariado $\mathrm{L}_{1,2}$ derivado da função K de Lotwick \& Silverman (1982) para testar a hipótese assumida para Completa Independência Espacial (CSI). Este estimador mede a dependência espacial entre dois padrões espaciais (p.e. se plântulas estão associadas positivamente

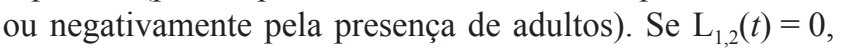
os padrões são independentes; valores positivos e negativos de $\mathrm{L}_{1,2}(\mathrm{t})$ indicam associação espacial positiva e negativa, respectivamente (De la Cruz et al. 2008).

Espacializando sobrevivência e mortalidade - As análises dos padrões espaciais de mortalidade e sobrevivência das plântulas em relação aos outros estádios de desenvolvimento (i.e. terceira hipótese) e perenes (i.e. quarta hipótese) foram realizadas com o modelo nulo de rotulagem independente desenvolvido por De la Cruz et al. (2008). Existem modelos nulos para muitas análises multivariadas de padrão de pontos, os quais são muito restritivos quanto ao tipo de pergunta que podem ajudar a resolver, porque não é possível testar se as associações positivas ou negativas entre os dois padrões (p.e. mortalidade e sobrevivência das plântulas) podem ser mediadas por um terceiro padrão (p.e. seca). Essa informação é útil para revelar os mecanismos que determinam as causas e os padrões espaciais e temporais da mortalidade de plântulas (De la Cruz et al. 2008). Para facilitar a visualização e interpretação dos resultados foi utilizada a forma linearizada da função $\mathrm{K}: \mathrm{L}\left(\mathrm{L}^{*}{ }_{12}[\mathrm{t}]=\left\{\mathrm{K}^{*}{ }_{12}[\mathrm{t}] / \pi\right\}^{1 / 2}-\mathrm{t}\right)$.

A quinta hipótese foi testada a partir da diferença na intensidade de agrupamento (i.e. se as plântulas mortas eram mais ou menos estruturadas que as plântulas vivas) com a diferença $K_{1}(t)-K_{2}(t)$. Para o mesmo fim foi testado se os pontos de cada padrão foram circundados por pontos de uma mesma marca com maior ou menor frequência do que o esperado com as diferenças $\mathrm{K}_{1}(\mathrm{t})-\mathrm{K}^{*}{ }_{1,2}(\mathrm{t})$ e $\mathrm{K}_{2}(\mathrm{t})-\mathrm{K}^{*}{ }_{1,2}(\mathrm{t})$. $\mathrm{O}$ efeito espécie-específico exercido por plantas adultas e perenes no destino de plântulas vivas e mortas de $M$. ernestii foi avaliado pela função bivariada $\mathrm{K}^{*}{ }_{1,2}$ (De la Cruz et al. 2008).

Em todos os testes realizados foram utilizados intervalos de confiança de 95\%. Estes foram construídos a partir dos maiores e menores valores da função K computada em 999 simulações do modelo nulo selecionado. Análises estatísticas e padrão espacial foram realizadas utilizando o software R ( R Development Core Team 2007).

\section{Resultados}

Aspectos demográficos - Foram detectados 686 indivíduos de $M$. ernestii em uma área de $1.025 \mathrm{~m}^{2}$ (densidade media total de 0,67 plantas $\mathrm{m}^{-2}$ ), com grande variação no número de indivíduos entre as parcelas estudadas. Esta variação tem uma forte relação com o grau de intemperismo dos afloramentos rochosos, 
evidenciada pelos valores da ANOVA: plântula $\left(\mathrm{F}_{3,634}=22,050, P=0,003\right)$, juvenis $\left(\mathrm{F}_{3,247}=17,775\right.$, $P=0,005)$, adultos $\left(\mathrm{F}_{3,470}=31,530, P=0,001\right)$ e todas as categorias combinadas $\left(\mathrm{F}_{3,1411}=61,907, P=0,0001\right)$. Em geral, as parcelas apresentaram maior número de indivíduos em afloramentos menos intemperizados (tabela 1).

Tabela 1. Valores médios do número de indivíduos entre os estádios de desenvolvimento encontrados nas nove parcelas. $(\mathrm{X})$ afloramento pouco intemperizado, $(\mathrm{Y})$ moderadamente intemperizado e $(\mathrm{Z})$ muito intemperizado $(\mathrm{CV}(\%)=$ coeficiente de variação).

Table 1. Mean values for the number of individuals among stages of development found in nine plots. (X) little weathered outcrop, $(\mathrm{Y})$ and moderately weathered $(\mathrm{Z})$ very weathered $(\mathrm{CV}(\%)=$ coefficient of variation $)$.

\begin{tabular}{ccrrl}
\hline Tratamentos & Plântula & Juvenil & Adulto & Todos \\
\hline X & $32,33^{\mathrm{a}}$ & $12,33^{\mathrm{a}}$ & $28,33^{\mathrm{a}}$ & $73,00^{\mathrm{a}}$ \\
$\mathrm{Y}$ & $8,33^{\mathrm{b}}$ & $3,33^{\mathrm{b}}$ & $7,33^{\mathrm{b}}$ & $17,00^{\mathrm{c}}$ \\
Z & $28,67^{\mathrm{a}}$ & $11,33^{\mathrm{a}}$ & $8,67^{\mathrm{b}}$ & $48,33^{\mathrm{b}}$ \\
Média Geral & 23,11 & 9,00 & 14,78 & 46,11 \\
$\mathrm{CV}(\%)$ & 55,94 & 54,81 & 79,55 & 60,87 \\
\hline
\end{tabular}

Letras diferentes sobrescritas na mesma linha indicam diferença estatística da média pelo teste de Tukey $(P<0,05)$.

Different superscript letters in the same line indicate statistically different means bay Tukey's test $(P<0.05)$.

Eventos de sobrevivência e mortalidade - Foram registrados 165 indivíduos de $M$. ernestii na parcela em 18 meses estudados, dos quais 117 morreram mais frequentemente na fase de plântulas $(75,90 \%)$, seguido por juvenis $(13,76 \%)$ e adultos $(10,34 \%)$. Das 136 plântulas georreferenciadas, 114 morreram, representando $83,82 \%$ do total.

As análises de Wilcoxon dos valores de mortalidade em relação à estação seca e chuvosa apresentaram maior ocorrência deste evento no período seco $(Z=-2,375$, $P=0,005)$. Não houve mudança na estrutura da população em relação aos adultos e juvenis, mas a flutuação dos valores de densidade foi devido ao número de plântulas na população recrutadas em diferentes momentos durante os meses de acompanhamento.

Os eventos de mortalidade ocorreram nas plântulas com diâmetro $\leq 30 \mathrm{~mm}$, como resultado das flutuações ambientais: meses consecutivos de baixa ou nenhuma precipitação ( $\mathrm{rs}=-0,753, P=0,007)$. O recrutamento de novas plântulas na população foi significativamente correlacionada com a umidade ( $\mathrm{rs}=0,817, P=0,002)$ e sempre ocorreu após pulsos de precipitação.

Padrão espacial univariado - A função K de Ripley calculada para todos os indivíduos de M. ernestii rejeitou a hipótese nula de Completa Aleatoriedade Espacial (CSR) em todas as parcelas (figura $1 \mathrm{a}-\mathrm{d}$ ), embora as intensidades sejam diferentes, com picos de agregação observados entre as escalas de distancias em todos os casos. Na parcela $\mathrm{G}$ o padrão aleatório foi evidenciado apenas acima de 2,00 m; as demais parcelas apresentaram padrão agregado em todas as escalas, mas com pico de agrupamento em $0,50 \mathrm{~m}$. A parcela $\mathrm{F}$, com ausência de plantas, não foi incorporada nas análises. As plantas nas parcelas $\mathrm{H}-\mathrm{L}$ foram dispostas em agregados em todas as escalas.

O padrão espacial do estádio adulto variou entre aleatório e agregado nas parcelas e escalas monitoradas (figura $1 \mathrm{e}-\mathrm{h}$ ). As plantas no estádio adulto na parcela $\mathrm{G}$ estavam agregadas entre $0,50-1,00 \mathrm{~m}$ e dispostas de modo aleatório nas demais escalas. Na parcela I os indivíduos adultos estavam distribuídos de forma aleatória nas escalas, no entanto nas parcelas $\mathrm{H} \mathrm{e} \mathrm{J} \mathrm{-} \mathrm{L}$ estavam agregados em todas as escalas.

A estruturação espacial do estádio juvenil (figura 1 i-l) se apresentou variável nas parcelas avaliadas: completamente aleatório na parcela $\mathrm{C}$ (figura $1 \mathrm{k}$ ) e com níveis de agregação apenas a pequena escala nas demais parcelas, com destaque para parcela D (figura 1 1) com maior intensidade de agregação que as demais. Esse padrão de agregação pode ser interpretado como perfil de agrupamentos evidentes, uma vez que a função, que é cumulativa, encontra cada vez mais indivíduos até $1,00 \mathrm{~m}$ e, após esta escala, a relativa ausência de indivíduos produz a queda na curva (figura 1 1). As parcelas E-H, com ausência de plantas neste estádio, inviabilizaram as simulações. Nas parcelas I - L a CSR foi rejeitada em todas as escalas.

O padrão espacial do estádio plântula, embora em outra escala e intensidade, foi predominantemente agregado (figura $1 \mathrm{~m}-\mathrm{p}$ ). Neste estádio, após a marca de 0,50 m de distância, há uma diminuição dos valores da função. Embora o padrão agregado esteja claro para as plântulas, o perfil de distribuição deste estádio é mais complexo, pois apresenta picos distintos de agrupamentos (figura $1 \mathrm{n}, \mathrm{p}$ ), o que representa núcleos distintos de agregação nestas parcelas. Nas demais parcelas (E, G-L) a hipótese CSR foi rejeitada em todas as escalas.

Quando analisamos as parcelas de dimensões maiores que $100 \mathrm{~m}^{2}(\mathrm{~J}, \mathrm{~K}$ e L) nos diferentes estádios de desenvolvimento de $M$. ernestii, a curva da função apresenta comportamento similar ao encontrado na 

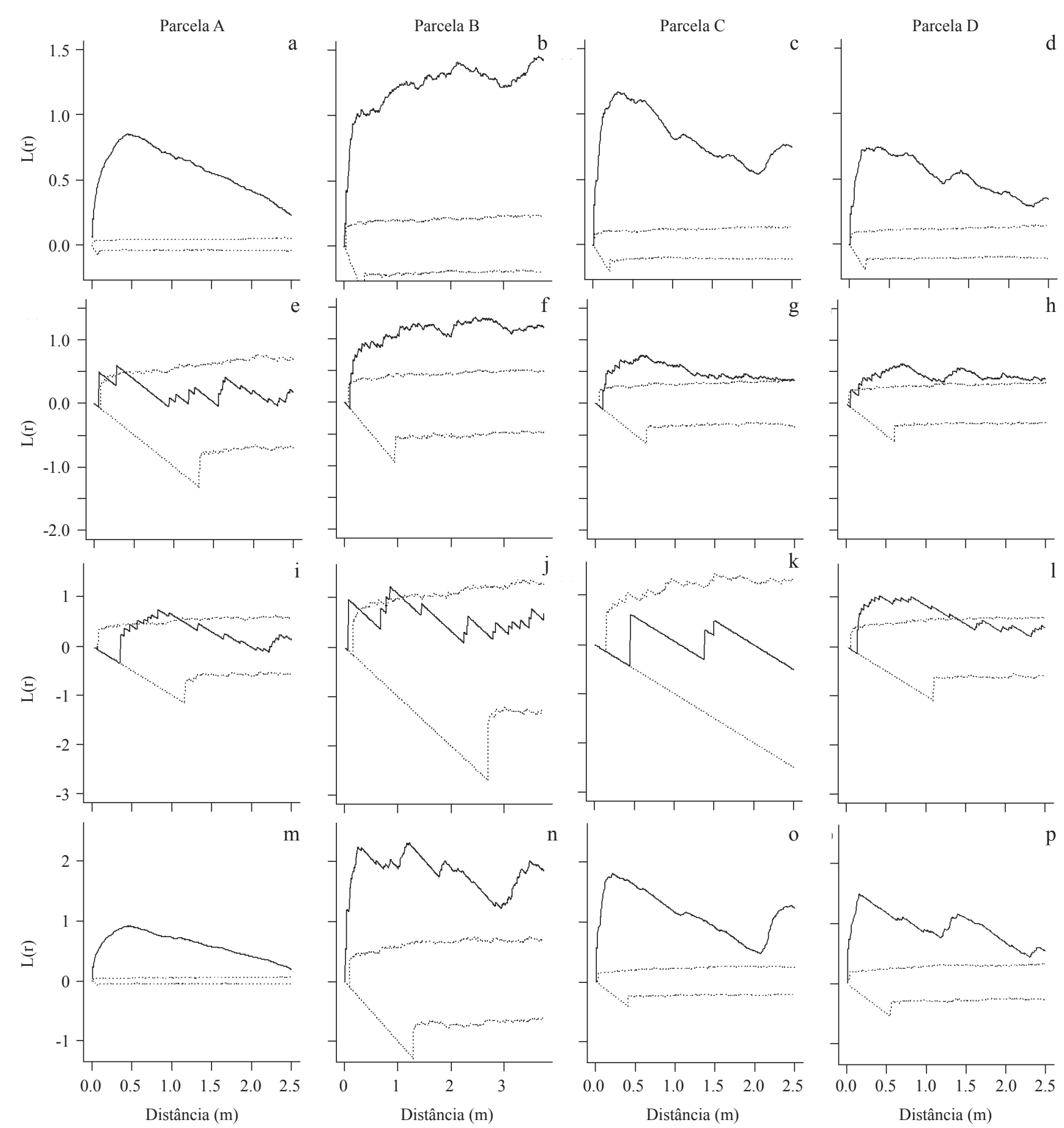

Figura 1. Padrão espacial de M. ernestii subsp. ernestii em diferentes parcelas e escalas, descrito pela função $\mathrm{L}_{(\mathrm{t})}$ : todos os indivíduos (a-d), adultos (e-h), juvenis (i-l) e plântulas (m-p). Linhas pontilhadas representam os limites dos intervalos de confiança para a hipótese de CSR, geradas a partir de 999 simulações. Linhas sólidas representam a função univariada $\mathrm{L}_{(\mathrm{t})}$ observada para círculos de $\mathrm{t}=2,5 \mathrm{~m}$ com $95 \%$ de significância. $\mathrm{O}$ valor de $\mathrm{L}_{(\mathrm{t})}$ acima do envelope de confiança indica agrupamento, valores dentro do envelope aleatoriedade, e valores abaixo do envelope indicam regularidade.

Figure 1. Spatial pattern of $M$. ernestii ssp. ernestii in different plots and scales, described by the function $\mathrm{L}_{(\mathrm{t})}$ : all individuals (a-d), adult (e-h), juveniles (i-l) and seedlings (m-p). Dashed lines represents the limits of the confidence intervals for the hypothesis of CSR, generated from 999 simulations. The solid lines represent the univariate function $\mathrm{L}_{(\mathrm{t})}$ observed for circles $\mathrm{t}=2.5 \mathrm{~m}$ with $95 \%$ significance. The value of $\mathrm{L}_{(\mathrm{t})}$ above the confidence envelope indicate clustering, values within the envelope of randomness, and below the envelope indicate regularity. 
Figura 1b, não sendo possível identificar em quais escalas os agrupamentos são mais evidentes.

Padrão Espacial Bivariado - As plântulas e adultos de $M$. ernestii estão associados positivamente entre 0-0,80 m nas parcelas analisadas, mas com diferença na intensidade de agrupamento (figura 2 a-d). Fato semelhante ocorre na relação entre plântulas e juvenis, mas com ausência de associação nas escalas abaixo de 0,40 m (figura 2 e, g-h), com exceção da parcela B (figura $2 \mathrm{f}$ ) com a hipótese de CSI aceita em todas as escalas. Os padrões entre juvenis e adultos apresentaram associação espacial em pequena escala (de 0 a $0,70 \mathrm{~m}$; figura $2 \mathrm{j}-1$ ), com exceção da parcela A, sem associação positiva a curta distância (figura 2 i). As demais parcelas analisadas apresentaram padrão similar ao encontrado nas parcelas demonstradas na figura 2, com exceção da parcela I, na qual a relação entre juvenis e adultos e entre
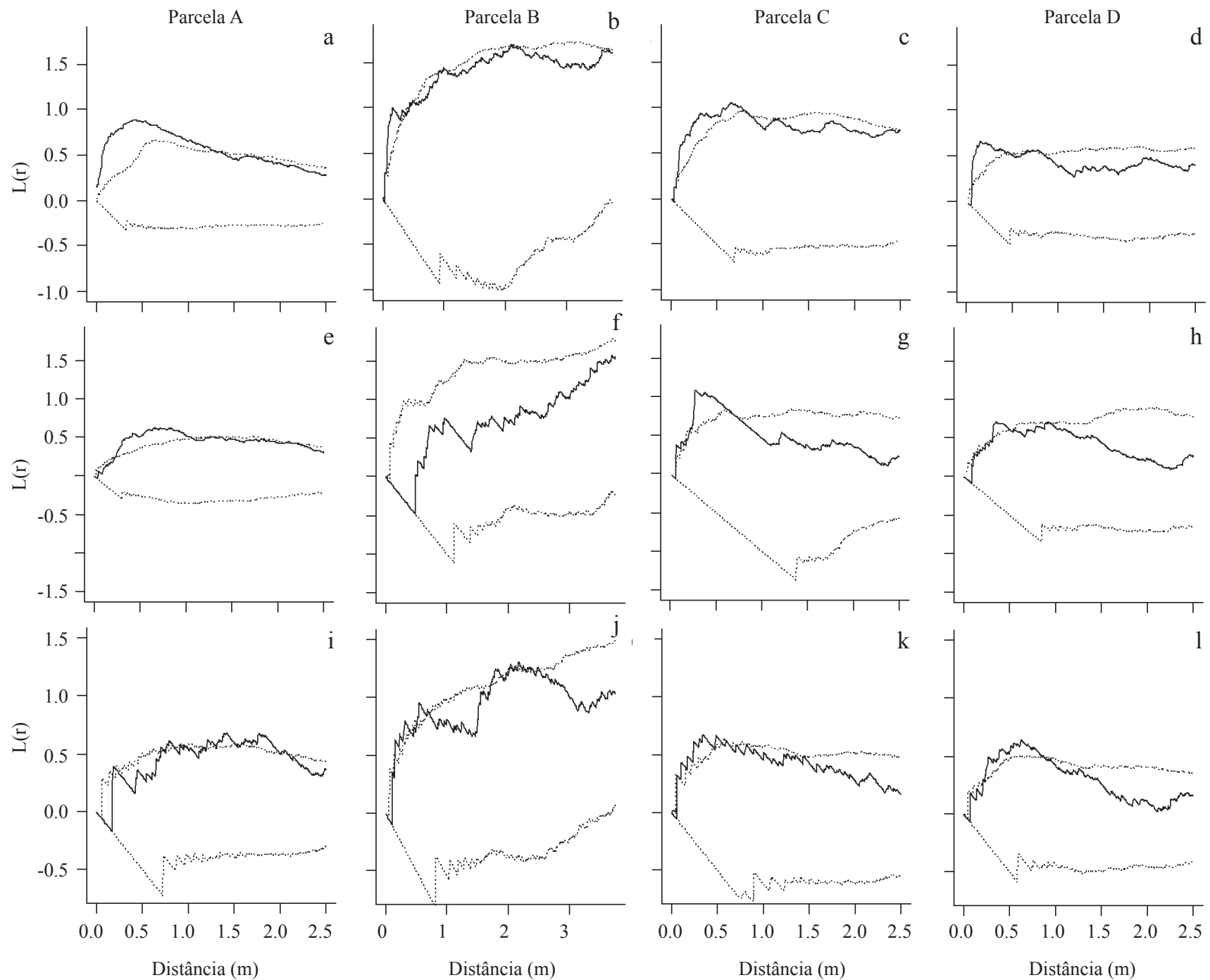

Figura 2. Padrão espacial de $M$. ernestii subsp. ernestii em diferentes escalas descrito pela função $\mathrm{L}_{1,2}$ : plântula com adultos (a-d), plântulas com juvenis (e-h) e juvenis com adultos (i-l). Linhas pontilhadas representam os limites dos intervalos de confiança para a hipótese de CSI, geradas a partir de 999 simulações. Linhas sólidas representam a função bivariada $\mathrm{L}_{1,2}$ observada para círculos de $\mathrm{t}=2,5 \mathrm{~m}$ com $95 \%$ de significância. $\mathrm{O}$ valor de $\mathrm{L}_{1,2}$ acima do envelope de confiança indica agrupamento, valores dentro do envelope aleatoriedade, e valores abaixo do envelope indicam regularidade.

Figure 2. Spatial pattern of M. ernestii ssp. ernestii at different scales described by the function $\mathrm{L}_{1,2}$ : seedling with adult (ad), juvenile with seedling (e-h) and juvenile with adults (i-l). Dotted lines represent the limits of the confidence intervals for the hypothesis of CSI, generated from 999 simulations. Solid lines represent the bivariate function $\mathrm{L}_{1,2}$ circles observed for $\mathrm{t}=2.5 \mathrm{~m}$ with $95 \%$ significance. The value of $\mathrm{L}_{1,2}$ above the confidence envelope indicate clustering, values within the envelope of randomness, and below the envelope indicate regularity. 
juvenis e plântulas permitiu que se aceitasse a hipótese de Completa Independência Espacial (CIS) em todas as escalas. As parcelas $\mathrm{J}-\mathrm{L}\left(>100 \mathrm{~m}^{2}\right)$ apresentaram pico de agregação entre 0 a 2,0 $\mathrm{m}$ e acima de $5 \mathrm{~m}$ para as análises bivariadas.

Não houve associação entre plantas adultas da M. ernestii georeferenciadas na parcela A e as demais perenes (figura 3 a-d). Padrão semelhante foi encontrado na relação entre juvenis (figura $3 \mathrm{~g}$-h), no entanto, apresentando associação positiva com Aspidosperma pyrifolium Mart. (Apocynaceae) entre 0,80-1,40 m (figura 3 e) e Jatropha ribifolia (Pohl) Baill. (Euphorbiaceae) em pequena escala (figura $3 \mathrm{f}$ ). As plântulas de $M$. ernestii apresentaram associação positiva à escala $0,20-0,50 \mathrm{~m}$ com Loasa rupestris Gard. (Loaseaceae) (figura 3 1) e a 1,25-2,20 m com Aechmea lingulata (L.) Baker (Bromeliaceae) (figura 3 1), mas não houve associação com A. pyrifolium e $J$. ribifolia (figura $3 \mathrm{i}-\mathrm{j}$ ).
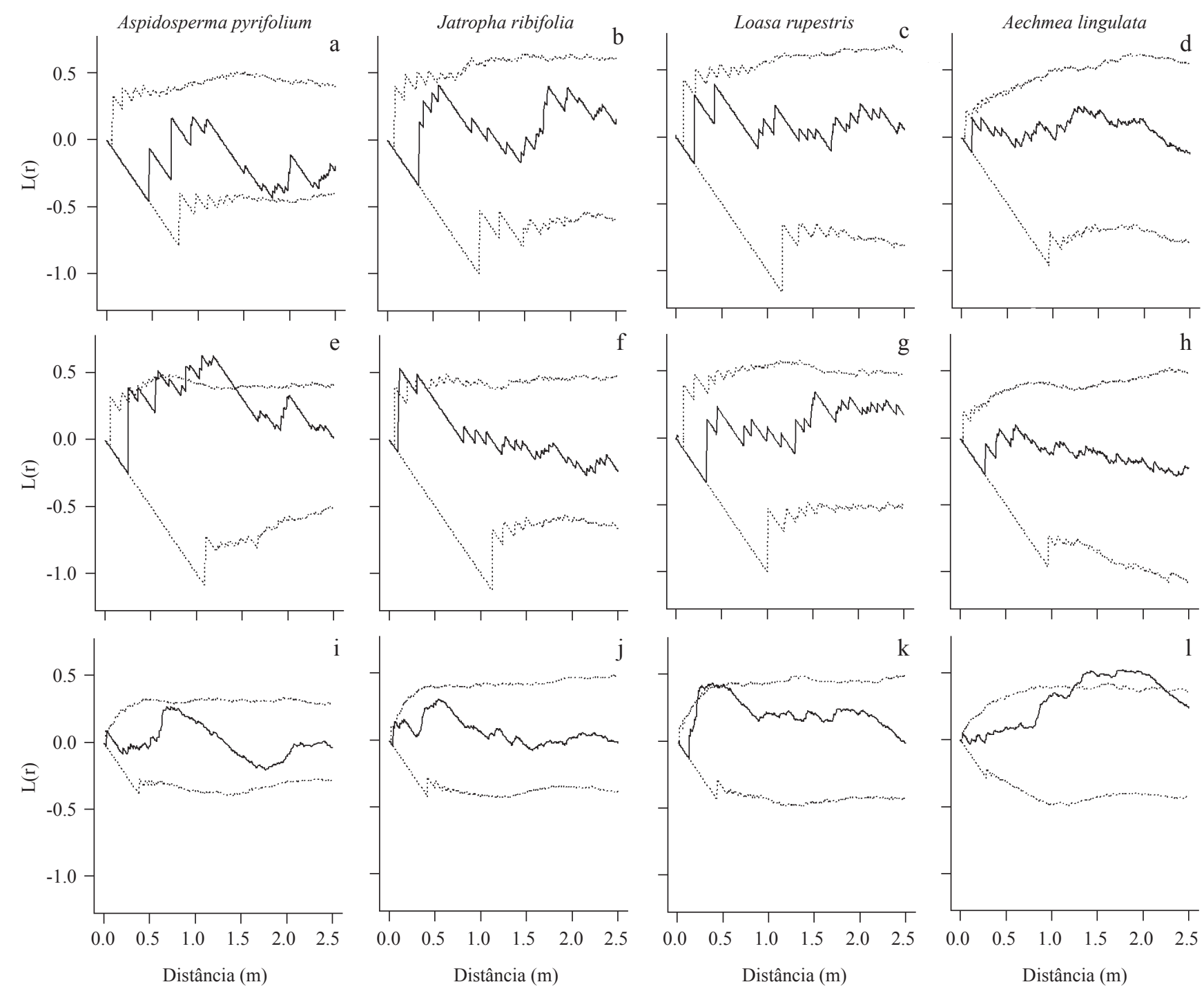

Figura 3. Padrão espacial, em diferentes escalas, descrito pela função $\mathrm{L}_{1,2}$ das plantas perenes A. pyrifolium (a, e, i), J. ribifolia (b, f, j), L. rupestris (c, g, k) e A. lingulata $(\mathrm{d}, \mathrm{h}, \mathrm{l})$ e dos estádios de desenvolvimento de $M$. ernestii subsp. ernestii amostrados na parcela A: adultos (a-d), juvenis (e-h) e plântulas (i-l). Linhas pontilhadas representam os limites dos intervalos de confiança para a hipótese de CSI, geradas a partir de 999 simulações. Restante da legenda como na Figura 2.

Figure 3. Spatial pattern at different scales, described by the function $\mathrm{L}_{1,2}$ of perennials A. pyrifolium (a, e, i), J. ribifolia (b, $\mathrm{f}$, 1), L. rupestris (c, g, $\mathrm{k})$ and $A$. lingulata $(\mathrm{d}, \mathrm{h}, \mathrm{l})$ and developmental stages of $M$. ernestii ssp. ernestii sampled in plot A: adults (a-d), juvenile (e-h) and seedlings (i-l). Dotted lines represent the limits of the confidence intervals for the hypothesis of CSI, generated from 999 simulations. Remainder of legend as in Figure 2. 
Espacialização dos Eventos de Mortalidade e Sobrevivência - A diferença entre o padrão de sobrevivência e mortalidade mostra que esta é mais agrupada que a primeira (i.e. diferença negativa; figura 4a). Por meio dos testes de segregação constatou-se que os índices não diferiram do esperado, portanto os casos de sobrevivência das plântulas de $M$. ernestii apresentam padrão aleatório em todas as escalas (figura 4b). Já os índices para a mortalidade das plântulas mostram que estas foram circundadas por plântulas mortas mais do que o esperado em todas as escalas (figura 4c), o que confirma a separação espacial de ambos os fenômenos (sobrevivência e mortalidade).

A associação espacial da sobrevivência e mortalidade de plântulas de M. ernestii com indivíduos
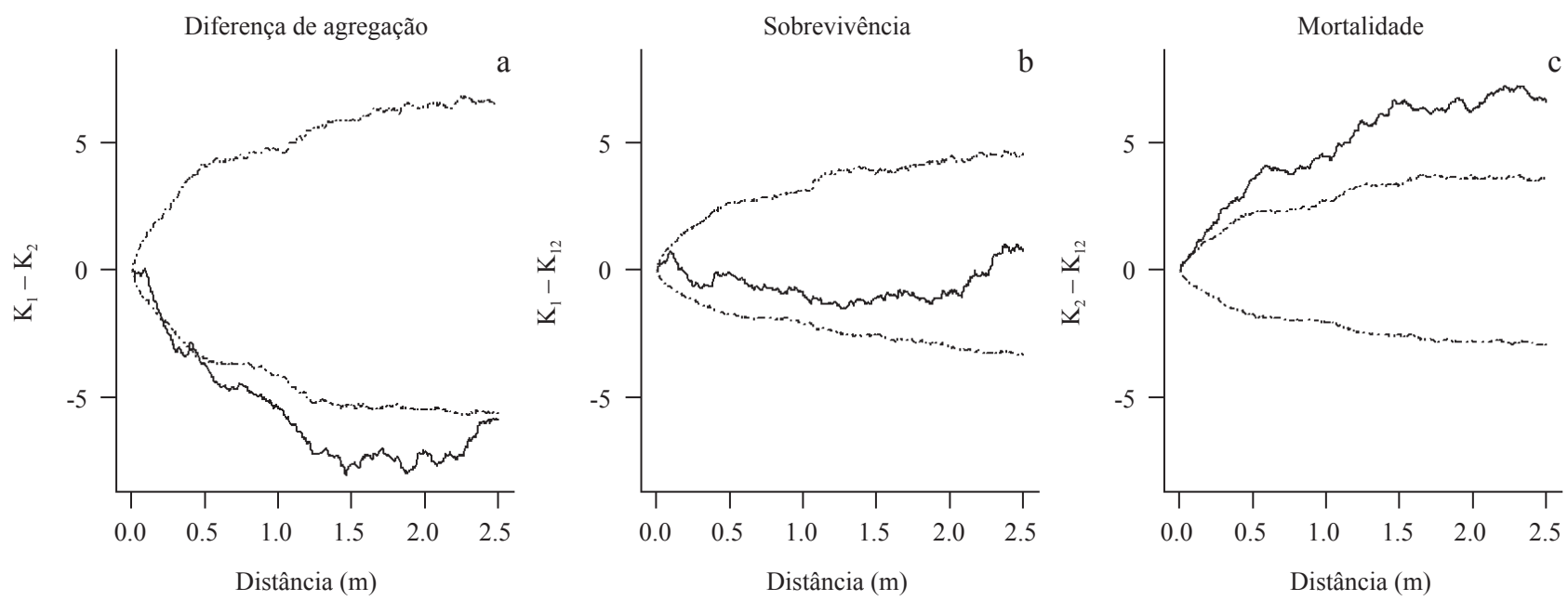

Figura 4. Diferença entre o padrão espacial de sobrevivência e mortalidade de plântulas de $M$. ernestii subsp. ernestii em um censo mensal de julho de 2007 a dezembro de $2008 . \mathrm{K}_{1}$ : função $\mathrm{K}$ univariada para o padrão de sobrevivência, $\mathrm{K}_{2}$ : função $\mathrm{K}$ univariada para o padrão de mortalidade, e $\mathrm{K}_{12}$ : função $\mathrm{K}$ bivariada para $\mathrm{o}$ padrão de sobrevivência e mortalidade. (a) Rejeição entre $\mathrm{K}_{1}$ e $\mathrm{K}_{2}$. (b). Aleatoriedade entre $\mathrm{K}_{1}$ e $\mathrm{K}_{12}$. (c) Agregação entre $\mathrm{K}_{2}$ e $\mathrm{K}_{12}$. Linhas sólidas representam a diferença e as linhas pontilhadas a significância da função do padrão de distribuição, computado com 999 simulações das marcas aleatórias "sobrevivência" e "mortalidade". Em todos os casos, a diferença esperada na hipótese nula de rotulagem aleatória é 0. Valores da função acima da linha superior do envelope de confiança indicam agrupamento, valores dentro do envelope aleatoriedade, e valores abaixo da linha inferior do envelope repulsão.

Figure 4. Difference between the spatial pattern of survival and mortality of seedlings of M. ernestii ssp. ernestii on a monthly census in july 2007 to december $2008 . \mathrm{K}_{1}$ : univariate $\mathrm{K}$ function for the pattern of survival, $\mathrm{K}_{2}$ : univariate $\mathrm{K}$ function for the pattern of mortality, and $\mathrm{K}_{12}$ : bivariate $\mathrm{K}$ function for the pattern survival and mortality. (a) Rejection of $\mathrm{K}_{1}$ and $\mathrm{K}_{2}$. (b) Randomness between $K_{1}$ and $K_{12}$. (c) Aggregation between $K_{2}$ and $K_{12}$. Solid line represents the observed difference and the dashed lines the significance of the standard distribution function, computed with 999 simulations of random marks "survival" and "mortality." In all cases, the expected difference in under the null hypothesis of random labeling is 0 . Function values above the upper confidence envelope indicate clustering, values within the envelope of randomness, and values below the lower envelope repulsion.

adultos da comunidade variou com a espécie de planta considerada (figura 5). A sobrevivência de plântulas apresentou associação negativa acima de $0,25 \mathrm{~m}$ com adultos e juvenis em conjunto (figura 5a), ao passo que a mortalidade teve associação positiva aproximadamente na mesma escala (figura 5b). Esta relação espacial e temporal se manteve quando associamos a sobrevivência e mortalidade de plântulas com o estádio adulto (figura 5c-d) e o estádio juvenil (figura 5e-f). A relação com as demais espécies perenes em conjunto não foi significativa (figura $5 \mathrm{~g}-\mathrm{h}$ ), o mesmo ocorrendo com $A$. pyrifolium (figura 5i-j) e A. lingulata (figura 5o-p). A sobrevivência de plântulas da $M$. ernestii foi associada positivamente com $J$. ribifolia a $0,40-0,50 \mathrm{~m}$ e 0,90 $1,70 \mathrm{~m}$ (figura $5 \mathrm{k}-1$ ) e com L. rupestris acima de $1,00 \mathrm{~m}$ (figura $5 \mathrm{~m}-\mathrm{n}$ ). 

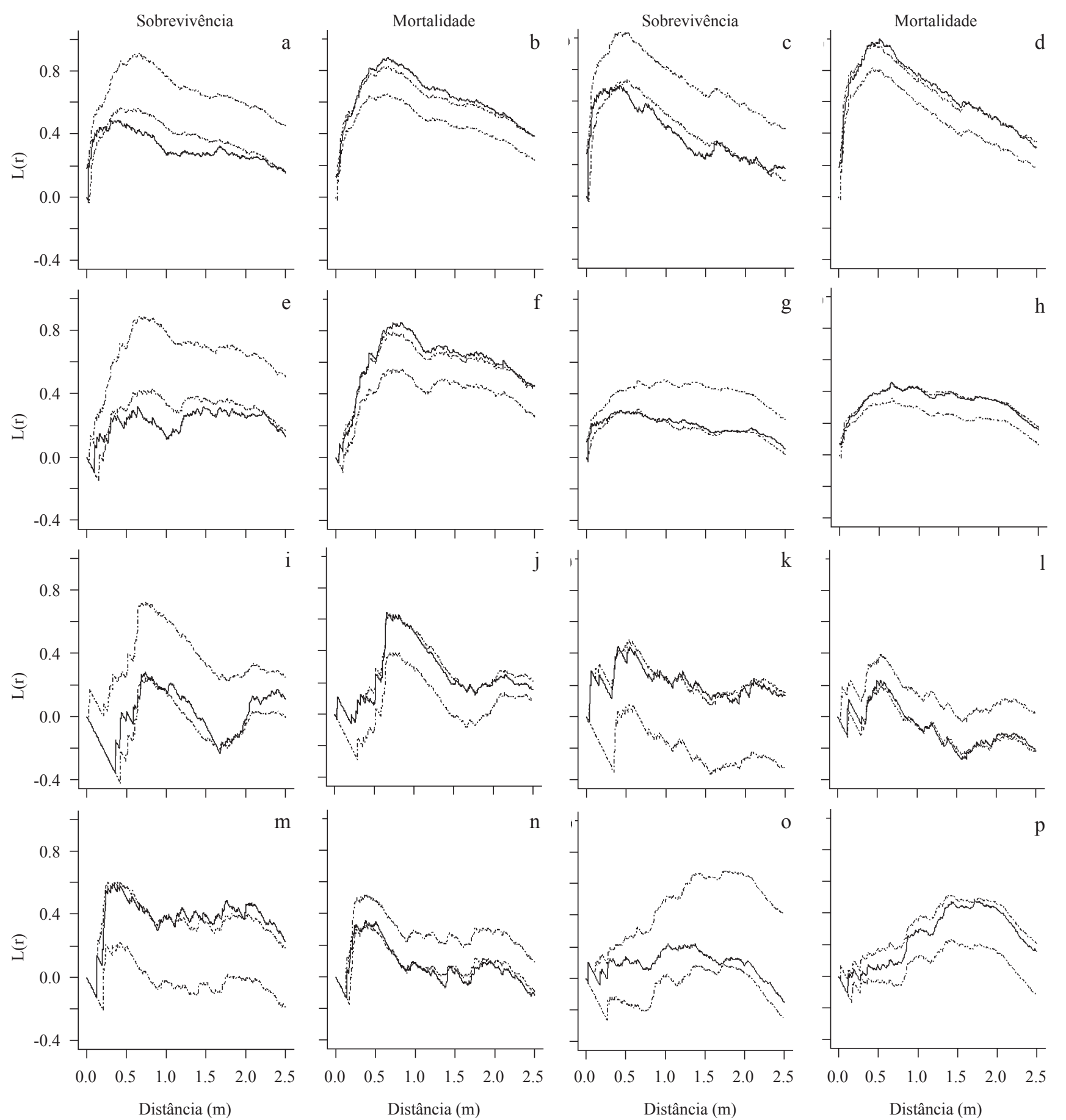

Figura 5. Mudanças temporais da relação espacial entre: adultos e juvenis (a-b), adultos (c-d), juvenis (e-f), todas as plantas perenes (g-h), A. pyrifolium (i-j), J. ribifolia (k-1), L. rupestris (m-n) e A. lingulata (o-p) com a sobrevivência e mortalidade de plântulas de $M$. ernestii subsp. ernestii. O valor significativo esperado na aceitação da hipótese de rotulagem independente ocorre nas escalas onde a função observada $\mathrm{L}_{12}$ não excede o envelope simulado.

Figure 5. Temporal shifts of the spatial relationship between: adult and juvenile (a-b), adults (c-d), juveniles (e-f), all perennials (g-h), A. pyrifolium (i-j), J. ribifolia (k-1), L. rupestris (m-n) and A. lingulata (o-p) and survival and mortality of M. ernestii ssp. ernestii seedlings. Significant departures from the expected values under the hypothesis of independent labeling occur at scales where the observed $\mathrm{L}_{12}$ function exceeds the simulated envelopes. 


\section{Discussão}

Fatores ambientais e demográficos - O estabelecimento de muitas espécies de plantas apresenta-se frequentemente sob plantas adultas de outras espécies (Ellner \& Shmida 1981), o que tem sido considerado um tipo de facilitação de grande importância na determinação da estrutura de comunidades de plantas de ambientes áridos (Callaway 2007). Desta forma, as sementes dispersas a longa distância podem ser ineficientes quando o ambiente mais favorável é localizado próximo à planta-mãe e a distribuição de ambientes similares é imprevisível (Ellner \& Shmida 1981, Escudero et al. 2005, Méndez et al. 2006, De la Cruz et al. 2008).

$\mathrm{O}$ elevado número de sementes produzidas e a sobrevivência de plântulas de cactos em períodos favoráveis conduz a um padrão de distribuição espacial agregado, como observado em espécies de zonas áridas e semi-áridas (Flores-Martínez et al. 1994, Leirana-Alcocer \& Parra-Tabla 1999). No entanto, isto não implica no incremento de indivíduos em populações naturais de $M$. ernestii, com valores elevados de mortalidade $(83,82 \%)$ em plântulas, fortemente influenciados pelos baixos valores de precipitação durante a estação seca (GodínezÁlvarez et al. 2005). Adicionalmente, os baixos valores de sobrevivência das plântulas com diâmetro $\leq 30 \mathrm{~mm}$ em $M$. ernestii determinam que a dinâmica populacional desta espécie dependa da sobrevivência dos adultos (Méndez et al. 2004).

O padrão de pulso de precipitação é o fator direcionador e dominante dos processos biológicos em escala temporal e espacial em ambientes áridos e semiáridos, afetando a dinâmica dos processos do solo e populações de plantas (Drezner \& Lazarus 2008). As mudanças na disponibilidade da água têm sido sugeridas como o mais importante fator mediador da facilitação e competição em ambientes semi-áridos (Maestre et al. 2005).

O estudo demográfico em $M$. ernestii detectou decréscimo da mortalidade na medida em que as plantas incorporam fitomassa e aumentam de tamanho, similar ao encontrado por Esparza-Olguín et al. (2002) para a cactácea Neobuxbaumia macrocephala (Weber ex Schum.) Dawson. Uma vez alcançado um diâmetro $>30 \mathrm{~mm}$, aumentam as chances de sobrevivência das plântulas em M. ernestii. Muitos cactos colunares (p.e. Neobuxbaumia Backeb, Carnegiea gigantea Britton \& Rose, Lophocereus schottii Englem.) apresentam valores muito elevados de mortalidade, próximo dos $100 \%$, durante os primeiros anos de vida (Valiente-Banuet \& Ezcurra 1991, Godínez-Álvarez \& Valiente-Banuet
2004). Ruedas et al. (2000) consideram que a diferença entre a densidade dos estádios de desenvolvimento pode indicar a ação dos efeitos estocásticos ambientais independentes da densidade, em fases específicas do ciclo de vida, bem como a influência dos microsítios no recrutamento de Mammillaria magnimamma Haworth., fatos estes observados em M. ernestii. Este processo aleatório termina por gerar distribuição desigual de indivíduos entre os estádios, a partir do recrutamento, após pulsos de precipitação geradores de condições ambientais favoráveis para germinação e estabelecimento (Mandujano et al. 2001, EsparzaOlguín et al. 2002). Estas condições específicas também foram relacionadas com combinações particulares de precipitação e temperatura para as espécies de cactáceas Carnegiea gigantea, Echinocactus horizonthalonius Lem., Ferocactus cylindraceus Engelm., Neobuxbaumia macrocephala e Opuntia echios Howell (Pierson \& Turner 1998, Hicks \& Mauchamp 2000, EsparzaOlguín et al. 2002, Godínez-Álvarez \& ValienteBanuet 2004).

M. ernestii apresenta estrutura populacional na qual o número de indivíduos decresce monotonicamente com o tamanho, o mesmo ocorrendo com as espécies de cactáceas Ferocactus acanthodes (Lem.) Britton \& Rose, Neobuxbaumia tetetzo (Coulter) Backeberg, e Opuntia echios (Jordan \& Nobel 1981, Godínez-Álvarez et al. 1999, Hicks \& Mauchamp 2000). Este tipo de estrutura populacional sugere crescimento e/ou manutenção da população, na qual os valores de mortalidade decrescem com o tamanho (Martínez-Ramos \& Álvarez-Buylla 1995).

Em populações com características demográficas adversas, tais como a baixa densidade populacional de indivíduos adultos e de plântulas sobreviventes, a ausência de reprodução clonal e dependência de indivíduos adultos para manutenção da estrutura etária representam os maiores riscos para o contingente de cactáceas (Hernández-Oria et al. 2007). As populações de $M$. ernestii têm sofrido transformações em função da destruição de habitats por atividades antrópicas e extrativismo predatório pelo potencial ornamental, os quais são impactantes para o contingente populacional das espécies do gênero Melocactus (Taylor \& Zappi 2004).

Estruturação espacial e eventos de sobrevivência e mortalidade na $M$. ernestii - Muitos fatores são responsáveis por determinar o padrão agregado dentro de populações de plantas. Os mais importantes são a heterogeneidade ambiental, o tipo de dispersão 
e a facilitação proporcionada por outras espécies (Escudero et al. 2005, De la Cruz et al. 2008). Avaliação em uma fina escala revelou que plântulas da M. ernestii emergem principalmente de forma agregada junto a indivíduos adultos e juvenis da espécie. Esta associação positiva pode ser interpretada pelo fenômeno de recrutamento agrupado dos diásporos, em função da dispersão a curta distância promovida pelo lagarto territorialista Tropidurus semitaeniatus (predominante em períodos secos) ou pela autocoria (predominante em períodos chuvosos), conferindo locais seguros para recrutamento (Hughes 2009). A avaliação destes fatores evidencia a importância da adaptação e colonização desta espécie em afloramentos rochosos (Escudero et al. 2005).

Plantas adultas de $M$. ernestii atuam de forma positiva no padrão espacial das plântulas co-específicas nos primeiros estádios de desenvolvimento (figura $3 \mathrm{a}$-d). Inversamente, as plantas perenes não apresentam nenhum tipo de associação com as plântulas de $M$. ernestii, com exceção de Loasa rupestris (figura 3k) e Aechmea lingulata (figura 31). Em ambientes áridos e semi-áridos a associação preferencial entre determinadas espécies de plantas já estabelecidas parece ser fundamental na germinação e no recrutamento de novos indivíduos nas populações de algumas espécies de cactáceas (Mandujano et al. 1998, Valiente-Banuet \& Ezcurra 1991). No entanto, a ocorrência de plântulas e juvenis de M. ernestii de forma isolada em fendas de rocha e sob blocos de pedra coaduna com as hipóteses de exclusividade de habitat e de facilitação por estruturas do microrrelevo (nurse objects), uma vez que a necessidade de uma planta berçário (nurse plant) nestas circunstâncias é altamente facultativa (Mandujano et al. 2002, López \& Valdivia 2007, Larrea-Alcázar \& Soriano 2008, Munguía-Rosas \& Sosa 2008).

As espacializações dos eventos de sobrevivência e mortalidade das plântulas de $M$. ernestii em função das plantas adultas co-específicas permitiram detectar uma associação espacial negativa entre o padrão de plântulas vivas e plantas adultas, mas associação positiva com outras espécies. Nossos resultados sustentam a idéia que a magnitude das interações bióticas em ambientes severos é espécie-específica (De la Cruz et al. 2008) e indicam que a associação positiva entre plantas adultas e plântulas sobreviventes (figure 2a-d) não é consistente a longo do tempo (figura $5 \mathrm{c}$ )

$\mathrm{O}$ padrão agregado das plântulas, relacionado à associação positiva da mortalidade com plantas adultas de $M$. ernestii, sustenta a hipótese de que a facilitação nos estádios iniciais de desenvolvimento provida pelo sítio materno não se sustenta após períodos de elevada escassez hídrica (De la Cruz et al. 2008). Estes padrões espaciais de sobrevivência e mortalidade configuram, em longo prazo, o padrão espacial da população adulta. No entanto, estas interações são dinâmicas e podem mudar dependendo do estádio do ciclo de vida, do grau da severidade abiótica (Valiente-Banuet \& Ezcurra 1991, De la Cruz et al. 2008) e do grau de intemperização do afloramento rochoso para as espécies exclusivas de habitat saxícola.

Arriaga et al. (1993) detectaram Jatropha vernicosa Brandegee, Haematoxylum brasiletto Karst. e Tecoma stans (L.) Juss. atuando como plantas berçário na cactácea Stenocereus thurberi (Engelm.) Buxbaum. Apenas as espécies perenes Loasa rupestris e Jatropha ribifolia mostraram efeito de facilitação sob as plântulas de M. ernestii durante os meses monitorados, com base no modelo nulo de rotulagem independente. Por outro lado, estas inferências podem estar representando apenas relações de co-existência destas espécies na dimensão do espaço em função dos elementos do microrrelevo. A relação de facilitação entre diferentes membros da comunidade vegetacional tem sido demonstrada por estudos em regiões que apresentam gradientes ambientais severos e estes efeitos podem depender da natureza específica da relação entre plântulas e plantas adultas (Flores \& Jurado 2003, Flores et al. 2004, Callaway 2007, Brooker et al. 2008, De la Cruz et al. 2008). Desse modo, estudos controlados devem ser conduzidos para melhor aproximação destas relações (Munguía-Rosas \& Sosa 2008).

Os processos que geram o padrão de distribuição espacial de indivíduos adultos e os padrões de distribuição espacial da sobrevivência e mortalidade de plântulas de $M$. ernestii são independentes. A associação negativa entre a sobrevivência de plântulas com plantas adultas e juvenis, e a associação positiva da mortalidade aproximadamente na mesma escala parece indicar que o território já ocupado pelos adultos é o mais apropriado para o estabelecimento das plântulas. A partir de $0,25 \mathrm{~m}$ de distância do centróide dos adultos e juvenis se encontram ambientes nos quais a sobrevivência se vê comprometida, o que não impede recrutamentos eventuais sob facilitação por elementos do microrrelevo (Flores \& Jurado 2003, Munguía-Rosas \& Sosa 2008).

Em populações naturais de $M$. ernestii a presença de associação positiva entre plântulas e indivíduos adultos sustenta o processo de facilitação intraespecífica nos primeiros estádios de desenvolvimento. O custo benefício emergente desta condição relativamente benigna é impulsionado, pelo menos em parte, pelo grau 
de estresse abiótico. Chu et al. (2008) consideram que a associação negativa da sobrevivência das plântulas com os adultos pressupõe efeitos de densidade nos eventos de mortalidade. No entanto esta circunstância não parece aplicável a $M$. ernestii, uma vez que cerca $90 \%$ das plântulas morrem por causa de secas sazonais, não havendo mortes adicionais além destes níveis.

A continuidade dos estudos envolvendo processos de facilitação em ambientes com severidade ambiental é necessária, assim como reconsiderações sobre a importância relativa de associações com nurse plants e nurse objects em cactos globulares, uma vez que estas associações são na grande maioria das vezes facultativas (Brooker et al. 2008, Munguía-Rosas \& Sosa 2008, Peters et al. 2008) e, em contraste com a associação nurse plant, os microambientes não competem por recursos (Resler et al. 2005).

Agradecimentos - Agradecemos à Dra. Denise Viola e a dois revisores anônimos pelas valiosas sugestões feitas ao manuscrito; ao Dr. Marlon Câmara Machado, Dr. Nigel Taylor e ao Dr. Abel Augusto Conceição, pela identificação das espécies vegetais; à bióloga Alice Ferreira da Silva Hughes, pelo suporte em campo, e ao apoio logístico do Sr. João Martins de Freitas, proprietário da Fazenda Recreio dos Freitas. Este trabalho foi financiado pela Fundação de Amparo a Pesquisa do Estado da Bahia (Fapesb: PPP-013/2006).

\section{Referências bibliográficas}

ANDERSON, E.F. 2001. The cactus family. Timber Press, Portland.

ARRIAGA, L.Y., MAYA, DIAZ, S. \& CANCINO, J. 1993. Association between cacti and nurse perennials in a heterogeneous tropical dry forest in northwestern Mexico. Journal of Vegetation Science 4:349-356.

BERTNESS, M. \& CALLAWAY, R.M. 1994. Positive interactions in communities. Trends in Ecology and Evolution 9:191-193.

BROOKER, R.W., MAESTRE, F.T., CALLAWAY, R.M., LORTIE, C.L., CAVIERES, L.A., KUNSTLER, G., LIANCOURT, P., TIELBÖRGER, K., TRAVIS, J.M.J., ANTHELME, F., ARMAS, C., COLL, L., CORCKET, E., DELZON, S., FOREY, E., KIKVIDZE, Z., OLOFSSON, J., PUGNAIRE, F., QUEIROZ, C.L., SACCONE, P., SCHIFFERS, K., SEIFAN, M., TOUZARD, B. \& MICHALET, R. 2008. Facilitation in plant communities: the past, the present, and the future. Journal of Ecology 96:18-34.

CALLAWAY, R. 2007. Positive interactions and interdependence in plant communities. Springer, Dordrecht.
CHU, C.J., MAESTRE, F.T., XIAO, S., WEINER, J., WANG, Y.S., DUAN, Z.-H. \& WANG, G. (2008), Balance between facilitation and resource competition determines biomass-density relationships in plant populations. Ecology Letters, 11:1189-1197.

COLAÇO, M.A.S., FONSECA, R.B. \& MACHADO, C.G. 2006. Biologia reprodutiva de Melocactus glaucescens Buining \& Brederoo e Melocactus paucispinus G.Heimen \& R.Paul (Cactaceae), no município de Morro do Chapéu, Bahia. Acta Botanica Brasilica 29:239-249.

DE LA CRUZ, M., ROMÃO, R.L., ESCUDERO, A. \& MAESTRE, F.T. 2008. Where do seedlings go? A spatiotemporal analysis of seedling mortality in a semi-arid gypsophyte. Ecography 31:1-11.

DREZNER, T.D. \& LAZARUS, B.L. 2008. The population dynamics of columnar and other cacti: a review. Geography Compass 2:1-29.

ELLNER, S. \& SHMIDA, A. 1981. Why are adaptations for long-range seed dispersal rare in desert plants? Oecologia 51:133-114.

ESCUDERO, A., ROMÃO, R.L., DE LA CRUZ, M. \& MAESTRE, F.T. 2005. Spatial pattern and neighbour effects on Helianthemum squamatum seedlings in a Mediterranean gypsum community. Journal of Vegetation Science 16:383-390.

ESPARZA-OLGUÍN, L., VALVERDE, T. \& VILCHISANAYA, E. 2002. Demographic analysis of a rare columnar cactus (Neobuxbaumia macrocephala) in the Tehuacan Valley, Mexico. Biological Conservation 103:249-259.

FLORES, J., BRIONES, O., FLORES, A. \& SÁNCHEZCOLÓN, S. 2004. Effect of predation and solar exposure on the emergence and survival of desert seedlings of contrasting life-forms. Journal of Arid Environments 58:1-18.

FLORES, J. \& JURADO, E. 2003. Are nurse-protege interactions more common among plants from arid environments? Journal Vegetation Science 14:911916.

FLORES-MARTINEZ, A., EZCURRA, E. \& SANCHEZCOLON, S. 1994. Effect of Neobuxbaumia tetetzo on growth and fecundity of its nurse plant Mimosa luisana. Journal of Ecology 82:325-330.

FONSECA, R.B.S., FUNCH, L.S. \& BORBA, E.L. 2008. Reproductive phenology of Melocactus (Cactaceae) species from Chapada Diamantina, Bahia, Brazil. Revista Brasileira de Botânica 31:237-244.

FRANCO, A.C. \& NOBEL, P.S. 1989. Effect of nurse plants on the microhabitat and growth of cacti. Journal of Ecology 77:870-886.

GODÍNEZ-ÁLVAREZ,H., RÍOS-CASANOVA,L.\& PÉREZ, F. 2005. Characteristics of seedling establishment of Stenocereus stellatus (Cactaceae) in the Tehuacán Valley, Mexico. The Southwestern Naturalist 50:375-407. 
GODÍNEZ-ÁLVAREZ, H. \& VALIENTE-BANUET, A. 2004. Demography of the columnar cactus Neobuxbaumia macrocephala: a comparative approach using population projection matrices. Plant Ecology 174:109-118.

GODÍNEZ-ÁLVAREZ, H., VALIENTE-BANUET, A. \& VALIENTE-BANUET, L. 1999. Biotic interactions and the population dynamics of the long-lived columnar cactus Neobuxbaumia tetetzo in the Tehuacán Valley, Mexico. Canadian Journal of Botany 77:203-208.

HERNÁNDEZ-ORIA, J.G., CHÁVEZ-MARTÍNEZ, R. \& SÁNCHEZ-MARTÍNEZ, E. 2007. Factores de riesgo em las Cactaceae amenazadas de uma región semiárida em el sur del desierto Chihuahuense, México. Interciencia 32:718-734.

HICKS, D.J. \& MAUCHAMP, A. 2000. Population structure and growth patterns of Opuntia echios var. gigantea along an elevational gradient in the Galapagos Islands. Biotropica 32:235-243.

HUGHES, F.M. 2009. Ecologia reprodutiva e morfometria de Melocactus ernestii (Vaupel) no município de Nova Itarana. Dissertação de mestrado, Universidade Estadual de Feira de Santana, Feira de Santana.

HUNT, D.R., TAYLOR, N.P. \& CHARLES, G. 2006. The newcactuslexicon. Volumes I. International Cactaceae Systematics Group. DH Books. Milborne Port.

IBGE - Instituto Brasileiro de Geografia e Estatística. 1993. Recursos naturais e meio ambiente: uma visão do Brasil. Departamento de Recursos naturais e Estudos Ambientais, Rio de Janeiro.

INMET. 2009. National Institute of Meteorology. www. inmet.gov.br (acesso em 12/01/2009).

IUCN. 2009. IUCN Red List of Threatened Species. http:// www.redlist.org (acesso em 28/04/2009).

JORDAN, P.W. \& NOBEL, P.S. 1981. Seedling establishment of Ferocactus acanthodes in relation to drought. Ecology 62:901-906.

JUÁREZ, R.I.N. \& LIU, W. 2001. FFT Analysis on NDVI annual cicle and climatic regionality in northeast Brazil. International Journal of Climatology 21:1803-1820.

LARREA-ALCÁZAR, D.M. \& SORIANO, P.J. 2008. Columnar cacti-shrub relationships in an Andean semiarid valley in western Venezuela. Plant Ecology 196:153-161.

LEIRANA-ALCOCER, J. \& PARRA-TABLA, V. 1999. Factors affecting the distribution, abundance and seedling survival of Mammillaria gaumeri, an endemic cactus of coastal Yucatán, México. Journal of Arid Environments 41:421-428.

LÓPEZ, R.P. \& VALDIVIA, S. 2007. The importance of shrub cover for four cactus species differing in growth form in an Andean semi-desert. Journal of Vegetation Science 18:263-270.

LOTWICK, H.W. \& SILVERMAN, B.W. 1982. Methods for analyzing spatial processes of several types of points. Journal of the Royal Statistical Society B Methodological 44:406-413.
MAESTRE, F.T., VALLADARES, F. \& REYNOLDS, J. 2005. Is the change of plant-plant interactions with abiotic stress predictable? A meta-analysis of field results in arid environments. Journal of Ecology 93:748-757.

MANDUJANO, M.C., MÉNDEZ, I. \& GOLUBOV, J. 1998. The relative contributions of sexual reproduction and clonal propagation in Opuntia rastrera from two habitats in the Chihuahuan Desert. Journal of Ecology 86:911-921.

MANDUJANO, M.C., MONTAÑA, C., FRANCO, M., GOLUBOV, J. \& FLORES-MARTÍNEZ, A. 2001. Integration of demographic annual variabilityin a clonal desert cactus. Ecology 82:344-359.

MANDUJANO, M.C., FLORES-MARTÍNEZ, A., GOLUBOV, J. \& EZCURRA, E. 2002. Spatial distribution of three globose cacti in relation to different nurse-plant canopies and bare areas. Southwestern Naturalist 47:162-168.

MARTÍNEZ-RAMOS, R. \& ÁLVAREZ-BUYLLA, E. 1995. Ecología de poblaciones de plantas en una selva húmeda de México. Boletín de la Sociedad Botánica de México 56:121-153.

MÉNDEZ, M., DORANTES, A., DZIB, G., ARGÁEZ, J. \& DURÁN, R. 2006. Germinación y establecimiento de Pterocereus gaumeri, uma cactácea columnar, rara y endêmica de Yucatán, México. Boletín de la Sociedad Botánica de México 79:33-41.

MÉNDEZ, M., DURAN, R. \& OLMSTED, I. 2004. Population dynamics of Pterocereus gaumeri, a rare and endemic columnar cactus of Mexico. Biotropica 36:492-504.

MMA. 2004. Biodiversidade da Caatinga: áreas e ações prioritárias para a conservação (J.M.C. Silva, M. Tabarelli, M.T. Fonseca \& L.V. Lins, org.). Ministério do Meio Ambiente, Brasília.

MUNGUÍA-ROSAS, M.A. \& SOSA, V.J. 2008. Nurse plants vs. nurse objects: effects of woody plants and rocky cavities on the recruitment of the Pilosocereus leucocephalus columnar cactus. Annals of Botany 101:175-185.

PETERS, E.M., MARTORELL, C. \& EZCURRA, E. 2008. Nurse rocks are more important than nurse plants in determining the distribution and establishment of globose cacti (Mammillaria) in the Tehuacán Valley, Mexico. Journal of Arid Environments 72:593-601.

PIERSON, E.A. \& TURNER, R.M. 1998. An 85-year study of saguaro Carnegiea gigantea demography. Ecology 79:2676-2693.

R DEVELOPMENT CORE TEAM 2007. R: a language and environment for statistical computing. - R Foundation for Statistical Computing.

RESLER, L.M., BUTLER, R.D. \& MALANSON, P.G. 2005. Topographic shelter and conifer establishment and mortality in an alpine environment, Glacier National Park, Montana. Physical Geography 26:112-125. 
REYES-OLIVAS, A., GARCÍA-MOYA, E. \& LÓPEZMATA, L. 2002. Cactishurb interactions in the coastal desert of northern Sinaloa, Mexico. Journal of Arid Environment 52:431-445.

RIPLEY, B.D. 1977. Modelling spatial patterns. Journal of the Royal Statistical Society B Methodological 39: $172-212$.

RITZ, C.M., MARTINS, L., MECKLENBURG, R., GOREMYKIN, V. \& HELLWIG, F. 2007. The molecular phylogeny of Rebutia (Cactaceae) and its allies demonstrates the influence of paleogeography on the evolution of South American mountain cacti. American Journal of Botany 94:1321-1332.
RIZZINI, C.T. 1982. Melocactus no Brasil. IBDF, Rio de Janeiro.

RUEDAS, M., VALVERDE, T. \& ARGÜERO, S.C. 2000. Respuesta germinativa y crecimiento temprano de plántulas de Mammillaria magnimamma (Cactaceae) bajo diferentes condiciones ambientales. Boletín de la Sociedad Botánica de México 66:25-35.

TAYLOR, N.P. \& ZAPPI, D.C. 2004. Cacti of eastern Brazil. Royal Botanic Gardens, Kew.

VALIENTE-BANUET, A. \& EZCURRA, E. 1991. Shade as a cause of the association between cactus Neobuxbaumia tetetzo and nurse plant Mimosa luisana in the Tehuacan Valley, Mexico. Journal of Ecology 79:961-971. 